\title{
La Investigación en la Práctica Artística como una Observación de Segundo orden.
}

Dr. Arturo Valencia Ramos. arturo.valencia@unison.mx Universidad de Sonora.

En este texto se evalúan las posibilidades de ubicación que ocupa la práctica artística en los espacios universitarios, particularmente en los mexicanos, en los cuales los procesos de acreditación obligan a profesionalizar y academizar al arte para posicionarse en un mismo nivel de diálogo y negociación con la ciencia. Retoma la experiencia de los países europeos que han incorporado la noción de Practice as Research en la enseñanza e investigación en las artes.

Tanto el arte como la educación son sistemas sociales funcionales, por lo que es desde esa perspectiva que abordamos nuestro objeto de estudio. La educación como sistema requiere estructuras organizativas y presupuestos teórico-ideológicos que orienten el sentido de sus acciones. Si esto es así en lo general, en lo particular las Instituciones de Educación Superior, IES, establecen criterios académico-normativos sobre los cuales se guían para operar. El orientador general de la educación es la profesionalización de los saberes, los cuales deben certificarse y acreditarse para obtener la validez oficial. De ahí la existencia de los organismos acreditadores que son contratados cuando se requiere validar la calidad de la educación ${ }^{1}$.

1 Con la misión de "contribuir al aseguramiento de la calidad y permanente mejora de los programas educativos en cualquiera de las disciplinas artísticas que ofrecen las instituciones de educación superior públicas y privadas de México, por medio de la evaluación y acreditación, así como llevar a cabo otras acciones que resulten en el beneficio de la formación artística profesional y el papel de las artes en la sociedad del conocimiento", hasta 2018 el CAESA ha acreditado
La acreditación de los saberes presupone también que quien los aprende tiene capacidad para ejercerlos. De ahí en parte también, cuando menos en el caso mexicano, que existan los trámites burocráticos para obtener una cédula profesional que garantiza ante los ojos de los demás que el portador de tal cédula tiene los conocimientos suficientes para ejercer la profesión. Sin embargo, y a pesar de eso, uno puede observar la existencia de prácticas cotidianas como las de los sanadores o curanderos que, sin estudios profesionales ni cédulas, ejercen la actividad sin ninguna garantía excepto la de la fé de quienes se someten a sus servicios ${ }^{2}$.

La educación superior, entonces, requiere ser acreditada para garantizar su calidad. Los criterios para tal acreditación varían según sea la profesión; sin embargo, nadie dudaría, por ejemplo, de los conocimientos que se transmiten a un estudiante de medicina en una facultad universitaria-que se discuta o no el nivel de calidad entre una universidad y otra no es un asunto normativo sino de valor. En este contexto, la educación privada aprovecha en nuestro país la cantidad de estudiantes rechazados en las universidades públicas para establecer su mercado y su posición ante la relación oferta/demanda.

\footnotetext{
$2.30 \%$ programas de Educación Artística; $4.60 \%$ de Gestión Cultural; $14.94 \%$ de Danza; $27.59 \%$ de Artes Visuales/Plásticas; $13.79 \%$ de Teatro; $36.78 \%$ de Música. Fuente: Consejo para la Acreditación de la Educación Superior de las Artes. caesa-artes.com

2 Aún más, es común encontrar sanadores o curanderos naturistas cuyo ejercicio no está normado, aunque sí es aceptado por los consumidores de productos naturales.
} 
El arte es un sistema social, ya lo hemos dicho, que opera en el medio del sentido, al igual que el sistema de la educación. Sin embargo, ningún artista requiere acreditarse para ser reconocido como tal. Los ejemplos en la música abundan, así como en las artes visuales. Pareciera que la transmisión de los saberes del arte puede darse por la tradición de manera informal en espacios extrauniversitarios y que es más bien su práctica lo que garantiza la calidad del creador y del ejecutante. ¿Dónde se puede observar si un músico es bueno? Seguramente el único lugar posible es en el escenario, o en algún otro espacio frente al público.

En las artes visuales, sin embargo, quien otorga el criterio de valor es el crítico o el curador (a veces es la misma persona), al incluir o excluir de una muestra la obra de uno o varios artistas. Esa garantía la precede los gustos propios del crítico-curador, así como las tendencias observables en el mercado del arte en cualquier nivel que se presente.

El presupuesto fundamental es que la educación superior profesionaliza los saberes y es la mayor garantía de la obtención y transmisión de conocimientos. Cuando la educación en las artes emerge o se incorpora a los sistemas organizativos de las IES requiere cartas de validación al igual que cualquier otra profesión como la medicina, el derecho o la economía; ha entrado al sistema por lo cual sus procesos y operaciones dejan de ser exclusivos. Más bien, su inclusión en el sistema obliga al arte a aceptar como propios los mecanismos de acreditación, o bien, adecuarse a ellos.

Podemos afirmar al respecto que las exigencias del sistema educativo a través de las instituciones de educación superior, como sistemas de organización, irritan al sistema del arte y lo conducen a producir operaciones por medio de las cuales propone respuestas para resolver la contingencia. La educación artística en el nivel superior debe entonces validar y acreditar su estatus en los espacios sociales que legitiman el estatus universitario de cualquier profesión. En este sentido lo que observamos es una interacción entre dos sistemas: El sistema del arte y el sistema de la educación. Este es el punto que debemos atender. Necesitamos observar los límites donde estos sistemas interactúan en los cuales además de presentarse procesos de irritación pudiéramos encontrar una suerte de acoplamiento estructural que define de manera permanente el ejercicio de las artes.

No vamos a discutir aquí que el sentido del arte, ya lo sabemos y damos por sentado, es la producción de obras cuyos criterios valorativos se presentan en la medida en que se constituyen como medios de comunicación simbólicamente generalizados: podemos distinguir una obra de arte de cualquier otro objeto, así sea éste un objeto de sentido común. Lo que vamos a discutir es ese punto de intersección entre el arte y la educación que se ubica en la producción de conocimiento; esto es en los espacios académicos de la investigación.

La postura teórica del texto es que el arte es un sistema social que se relaciona con el entorno y al establecer esas relaciones se define. En este sentido el sistema educativo forma parte del entorno del arte. Por esta razón toda investigación que se aborde requiere considerar estas condiciones para el desarrollo de la observación como mecanismo descriptivo y analítico. Esto implica que el abordaje y la posición del observador determinan el tipo de investigación que se emprende.

Tampoco queremos discutir aquí cualquier forma de investigación externa al sistema del arte, lo que hasta ahora y tradicionalmente se ha entendido como investigación sobre el arte, una operación externa, propia de las ciencias sociales y humanidades, entre otras, que lo abordan como objeto de estudio con un sujeto externo a su desarrollo-y que ha desplazado cualquier interés epistemológico del 
artista, como investigador, por lo que se ubican sus hallazgos en un nivel subordinado.

El abordaje de la investigación desde las artes la tenemos que ubicar en diferentes niveles dentro de nuestras IES dado que, como resulta obvio, la exigencia al respecto varía entre los niveles de licenciatura, maestría y doctorado-o cualquier otra forma de validación de pregrado y posgrado. Sin embargo, lo que debemos definir y hacer explícita es la práctica de la investigación, así como los productos esperados como resultado de tal práctica.

Al ser el sistema del arte un sistema complejo, la obra se aborda como un medio de comunicación simbólicamente generalizado, por lo cual el interés de la investigación en este sentido se puede orientar hacia la capacidad comunicativa del medio, incluyendo los aspectos técnicos y metodológicos, así como a las posibilidades de duplicación de la realidad; dicho, en otros términos: la investigación, al ser una observación de segundo orden atiende tanto la contingencia como la reducción de complejidad.

Estamos hablando, entonces, de la práctica artística como investigación, que se va a especificar según el tema a abordar y la perspectiva de la observación. Pero este no es un asunto abstracto, sino que se concretiza en el espacio social de la investigación, así como a partir de quien investiga. La especificación funcional recae así, en principio, en el docente y en las condiciones propias de la investigación. Una vez hecha esta especificación, los segundos involucrados son los estudiantes en sus espacios académicos.

Cuando me refiero a la investigación desde las artes, me estoy refiriendo a la que se desarrolla desde el interior mismo de la práctica artística y cuyos resultados se observan en la propia obra producida. Así que introduciré a partir de aquí el término investigación artística para diferenciarlo de otras formas de investi-

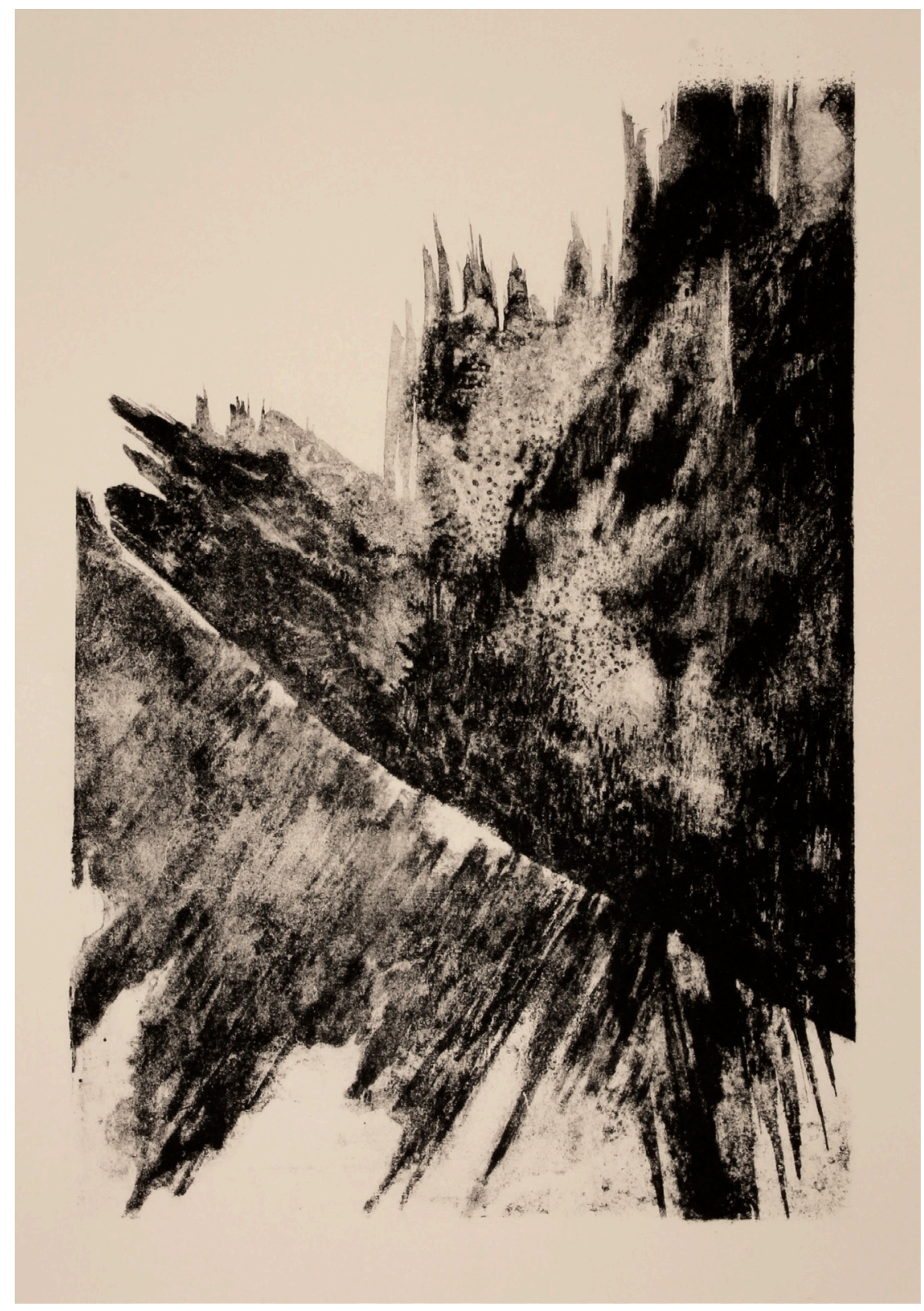

gación que tienen al arte como "objeto". Esto no implica la exclusión de métodos Litografía. Adriana Salazar investigativos propios de las ciencias sociales y humanidades, sino la incorporación de metodologías susceptibles de adaptarse a las condiciones de la práctica artística, sin que esta se subordine a los requerimientos metodológicos.

Por esta razón propongo el aprendizaje y utilización de metodologías cualitativas para la investigación tales como las que se derivan de la Teoría 
Fundamentada y de la Teoría de Sistemas Sociales, cuyos enfoques posibilitan la re-entrada del investigador en el fenómeno investigado-la práctica artística. De esta manera se pueden obtener dos tipos de productos: uno que de cuenta del proceso a validarse con artículos y ensayos, es decir los medios de comunicación simbólicamente generalizados del sistema de la ciencia ${ }^{3}$; y el otro, la obra, como medio de comunicación simbólicamente generalizado del sistema del arte. Se produce así, en términos de conocimientos y en términos estéticos.

En este sentido la propuesta es abordar aspectos epistemológicos de las teorías, poniendo énfasis en la metodología. Sin embargo, la contextualización propia de la metodología cualitativa no se propone aquí desde la perspectiva del sujeto frente al objeto, sino en el establecimiento de distinciones cuyos procedimientos se aseguran por medio de operaciones de observación de segundo orden, una vez que se han establecido las dimensiones objetual, social y temporal.

Aquí hemos asumido que la particularidad distintiva del arte como sistema es que la observación de segundo orden se produce en el plano de lo perceptible. Si esto es así, parece entonces que lo que caracteriza a las obras de arte es su capacidad de comunicar a partir de operaciones propias de la percepción y la conciencia, que trascienden con mucho la mera función representativa o mimética que hasta ahora se les ha concedido. De ahí que requiere formas particulares y específicas de investigación.

Una vez establecido que tanto la ciencia como el arte son sistemas sociales, aquí reconocemos que la observación de segundo orden no forma parte únicamen3 El discurso académico está constituido por el conjunto de textos producidos por la comunidad a través de medios científicos y académicos tales como publicaciones periódicas, libros especializados, conferencias en congresos de la disciplina, entre otros. Otro criterio de delimitación tiene una base epistemológica: el discurso académico se caracteriza por la producción de conocimiento (Federico Navarro y Ana Brown, Lectura y Escritura de Géneros Académicos, en Alzari, 2014, p. 76). te de la estructuración del conocimiento científico, sino que informa también operaciones particulares de observación en el arte. Por esta razón el propósito fundamental de este trabajo es mostrar que la autorreferencialidad del arte es una operación de diferenciación funcional cuyas comunicaciones se pueden investigar.

La investigación, de esta manera, forma parte de las operaciones del sistema del arte, por lo cual no debería sorprendernos que sus productos, además de ubicarse en el campo de la estética, pueden ubicarse en el campo de otros sistemas sociales. En este sentido, la práctica artística es también una operación investigativa que no se reduce al placer estético, sino que se contextualiza en diversos espacios del mundo social; es decir, del entorno del propio sistema del arte.

¿Por qué la investigación cualitativa? porque "se enfoca a comprender y profundizar los fenómenos, explorándolos desde la perspectiva de los participantes en un ambiente natural y en relación con el contexto" (Hernández Sampieri, 2010, p. 364), que es justamente el interés del artista en su práctica cotidiana cuando pretende mostrarnos "la realidad", además de que la autoreferencialidad queda especificada en esa práctica ${ }^{4}$.

En efecto, la investigación orientada por la práctica actualmente se ha relacionado con métodos cualitativos. Si bien es cierto que en los países del primer mundo esto ha sido posible, en nuestros países latinoamericanos es aún emergente y contamos con poca experiencia al respecto. Por esa razón aquí nos orientamos solo a la teoría fundamentada dado que se trata de poner énfasis en la construcción de la teoría ${ }^{5}$.

4 La autorreferencialidad no significa aqui una acción nihilista o solipsista del artista (cosa por demás frecuente), sino la referencia del sistema del arte y sus operaciones.

5 Currently there is a strong alignment between practice-led research and some research strategies and methods from qualitative tradition. For example, reflective practice, action research, grounded theory and participant-observation all inform practitioner-research generally. However, the important point to make is that practice-led research cannot merely be subsumed under the qualitative research framework. Practice-led 
Al ser el arte un sistema social, se producen observaciones de segundo orden que le son propias de sus operaciones. $\mathrm{Al}$ ser la obra de arte un medio de comunicación simbólicamente generalizado, la comunicación se ubica como unidad de análisis. También por esta razón la teoría de sistemas sociales permite un acercamiento más preciso cuando se trata de la investigación artística ${ }^{6}$. La diferenciación sistémica implica que el arte se ha diferenciado de su entorno, y la diferenciación funcional implica que ha distinguido su función de otras, incluso al interior del sistema.

La investigación artística así se especifica para la producción. Podemos distinguir del sistema de las artes, las artes visuales, y de ellas, la pintura, la escultura o el grabado-solo por citar esos ejemplos. De esta manera al investigar hemos orientado nuestro interés en principio por la producción de obras de arte y de manera colateral por la producción de conocimiento. Los programas de estudio de nuestras IES incluyen el espacio académico de Investigación solo que hasta ahora - y es ya un lugar común decirlo-hemos orientado nuestra metodología desde la ciencias sociales y humanidades. En este mismo texto lo estamos haciendo a efecto de trazar la distinción arte/ciencia y a partir de ahí generar nuestros métodos propios como artistas, docentes e investigadores.

La experiencia reciente en el Programa de Licenciatura en Artes Plásticas donde imparto el espacio académico de Seminario en Artes Plásticas ha permitido reorientar research employs its own distinctive research approach with its own strategies and methods, drawn from the long-standing and accepted working methods and practices of artists and practitioners across the arts and emerging creative disciplines (Brad Haseman, Rupture and recognition: Identifying the performative research paradigm, en Barrett, 2007, pp. 3306, versión Kindle).

6 Es común encontrar una vinculación de la teoría de sistemas sociales (en adelante TSS) con las metodologías y técnicas de investigación cualitativas. Esto, fundamentalmente, a causa de la centralidad de dos conceptos centrales en la propuesta teórica de Niklas Luhmann: la 'observación de segundo orden' como herramienta metodológica y la 'comunicación' como unidad de análisis (Urquiza, 2017, p. 22). el sentido de la investigación. Hoy estamos investigando para crear obras, sin embargo, seguimos un protocolo transdisciplinar por medio del seguimiento de una bitácora en la que los estudiantes trazan su ruta de trabajo y van fundamentando, en la medida en que avanzan, su observación-y, por ende, la posibilidad de generar teoría.

El propósito de nuestra práctica al hacer investigación artística es cubrir en su totalidad los aspectos académicos que nos acrediten en el nivel de licenciatura: el estudiante no solo debe crear obras de arte, sino generar conocimiento dado que además de ser artista se espera que sea docente e investigador. He aquí la diferencia fundamental. En efecto ningún artista requiere acreditarse para ser reconocido como tal; sin embargo, un egresado de un programa de licenciatura sí requiere contar con los créditos suficientes y necesarios para ejercer sus conocimientos en la docencia y en la investigación.

Hacemos énfasis en la observación de segundo orden como método ya que así podemos ubicar al estudiante en su perspectiva de observación y evitar imponerle alguna-además de la que tradicionalmente le hemos impuesto desde las ciencias sociales. Al observar el entorno lo tematiza o puede tematizarlo de forma tal que al percibir los objetos y las operaciones los transformaría en obras de arte, es decir podría transfigurar, o lo que, es más, duplicar la realidad. Si la realidad es comunidad o comunicación, entonces está creando nuevas realidades que se incorporarán tanto al sistema del arte como a su entorno ${ }^{7}$ : ha creado algo nuevo dentro de las operaciones autopoiéticas del sistema, pero no solo eso, sino que ha producido conocimiento.

El estudiante también establecerá distin-

7 In the process of invention the heterogeneous interests of the poet, the choreographer, the hip-hop deejay, the AutoCad designer and the landscape architect display their common interest. The condition of invention - the state of being that allows a state of becoming to emerge-is a perception, or recognition, of the ambiguity of appearences (Paul Carter, Interest: The Ethic of Invention, en Barrett, 2007, pp. pos. 437, versión Kindle). 


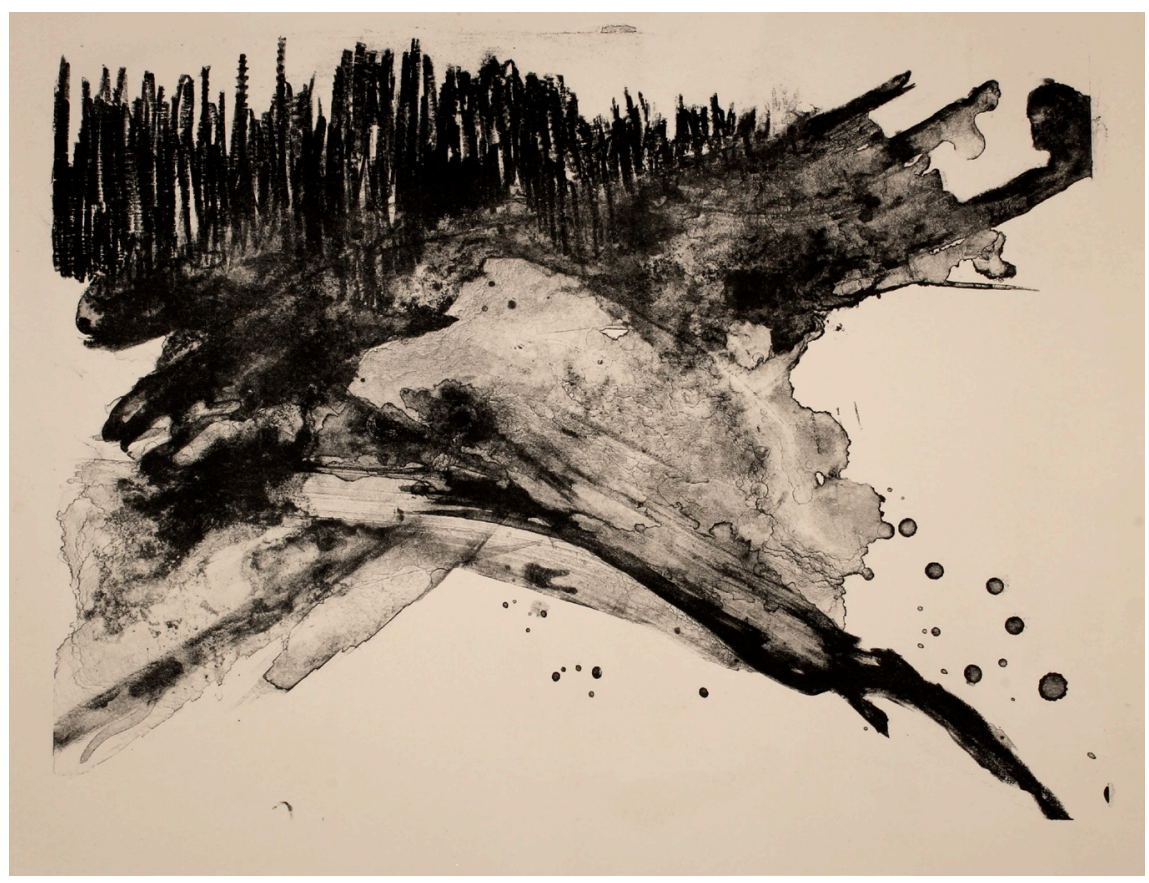

Litografía. Adriana Salazar ciones guía para producir datos cualitativos con los cuales trabajará a lo largo de su proceso creativo. Esas distinciones pueden presentarse dentro de la producción misma de la obra (utilizar ciertas técnicas en lugar de otras, ciertos materiales en lugar de otros), o bien, en su entorno social del cual tematiza para producir. La complejidad del arte contemporáneo nos obliga a esa forma de observación ya que, como decimos, no podemos observar todo de manera sincrónica, sino que tenemos que construir a partir de lo observado-con todos los riesgos que implica el punto ciego de la observación. En pocas palabras utilizará el método de análisis comparativo constante para poder construir la realidad.

Cuando proponemos la Teoría Fundamentada lo hacemos porque su base es la fundamentación de los conceptos obtenidos de los datos empíricos y porque el artista tiene como divisa principal el ser creativo y crítico. Por tanto, es de esperar que en su desarrollo ulterior, sea como artista o profesional del arte, aplique el método de manera crítica y así enriquezca la realidad con sus producciones, a la vez que pueda clarificar las similitudes y diferencias entre la ciencia y el arte, así como los valores cognitivos de ambas - a pesar de los desacuerdos que puedan existir al respecto ${ }^{8}$.

La complejidad del arte, lo hemos señaldo anteriormente, implica también el establecimiento de relaciones y redes de intercambio duraderos que posibilitan el desarrollo tanto de los académicos como de los artistas en su práctica. Ya no es posible el solipsismo, a pesar de su existencia, puesto que esa complejidad requiere de constantes comunicaciones que, si bien es cierto, en los países desarrollados es ya una práctica común, en los nuestros emerge y madura en la medida de la profesionalización de las artes 9 .

Ahora bien, también es de esperar que el estudiante continúe su trayectoria académica y pueda desarrollar investigación en niveles superiores cuando, en realidad, ya ha cubierto la cuota de producción artística en la licenciatura o, dicho en otros términos, cuando ya tiene claro que además de producir obra, puede profundizar en el conocimiento del arte desde cualquier perspectiva. La experiencia en el Posgradio en Humanidades de la Universidad de Sonora en el cual están inscritos tanto creadores como ejecutantes y han presen-

\footnotetext{
8 Advocates and opponents of the view that art has cognitive value tend to agree on one point. They agree that, if art is a source of knowledge, it is so in the same way as science is. Since science has been the paradigm of inquiry for centuries, this agreement is not surprising. Nevertheless, although both art and science can contribute to our knowledge, they do so in radically different ways. Each of these fundamentally distinct forms of inquiry corresponds to a basic sort of representation. Semantic representations (employed in the sciences) help us understand the world in a way very different from the way illustrations (characteristic of the arts) do (Young, 2001, p. 65).
}

9 Within the last two years, the country's cultural professionals have been witnessing a remarkable surge of interest in policy thinking and policy research in their field. To these professionals, who work in and worry about the arts and humanities, this interest in policy is remarkable because it is so rapid, so strenuous, and so promising. A long-standing policy vacuum has existed in U.S. cultural life. Today, there is a growing acknowledgment among scholars and practitioners that policy discussion, articulation, research, and action are important to the future of the arts and humanities (Alberta Arthurs, Arts Research: From the Hill to the 'Hood, en Morris, 2003, p. 31). 
tado proyectos de investigación artística sin que eso haya implicado el abandono de la práctica.

La experiencia del profesor Jan Kaila en Helsinski es ilustrativa de este proceso y nos puede servir como un buen ejemplo de las posibilidades reales que tienen los maestrantes y doctorantes de trascender en sus disciplinas específicas. Cuando aplicó al programa de doctorado en 1997 esperaba que la academia tuviera una visión clara de la investigación en las artes visuales, que la considerara como un todo en el cual el trabajo creativo, la producción artística, fuera lo más relevante y que las preguntas de investigación surgieran de esa producción, sin considerar que tal investigación debiera cubrir todos los requerimientos de la ciencia. Se trataba, en pocas palabras, demostrar las habilidades artísticas así como el conocimiento académico $^{10}$.

Las preguntas sobre las posibilidades de la investigación artística quedan, sin embargo ahí para resolver en la práctica: ¿contribuimos los artistas con nuestras investigaciones al desarrollo del arte, o es un mero requisito académico que debemos cubrir en nuestra trayectoria? Yo aseguro que sí contribuimos a su desarrollo, aunque se puedan plantear muchas preguntas sobre las posibilidades de una

10 (Jan Kaila, What is the point of research and doctoral studies in art?, en Balkema, 2004, p. 59) epistemología del arte ${ }^{11}$.

Yo no estaría tan seguro, como algunos lo pueden proponer, que los cambios en las curricula de la educación artística correspondan a una crisis del arte contemporáneo. Más bien, estoy seguro de que la diversificación en las artes, sus especificaciones funcionales, requieren de nuevas formas de observación desde la complejidad hacia la complejidad, mismas que deben partir desde los espacios académicos. No creo que sea posible de otro modo, si en verdad queremos desarrollarnos con relación al sistema del arte. Podemos ser escépticos en muchos sentidos, pero también, y es lo más, tenemos las herramientas y los recursos para ser optimistas en que al final del día el arte como sistema logrará posicionarse en el territorio del conocimiento, no como un anexo más de la ciencia, sino en el mismo nivel jerárquico de producción epistemológica.

11 A much more difficult question relates to whether artistic research has something to contribute to artists or others interested in the subject, something that the already existing research, institutions or art world are unable to provide. I think it has. Artistic research at best involves going in depth into the special knowledge involved in artistic authorship and distributing it, both to the visual art field and possibly elsewhere, and only an investigative artist can do this. In practice, this distribution takes place via exhibitions, publications and teaching that result from artistic research (Kaila, 2004, p. 65)

\section{Referencias:}

Alzari, I., et al. (2014). Manual de Escritura para Carreras de Humanidades. Buenos Aires: Editorial de la Facultad de Filosofía y Letras. Universidad de Buenos Aires.

Balkema, A. W. S., Henk (Editors). (2004). Artistic Research. Amsterdam: Rodopi.

Barrett, E. B., Barbara. (2007). Practice as research: Approaches to creative arts enquiry. New York.

Hernández Sampieri, R., et al. (2010). Metodología de la Investigación. México: McGraw Hill / Interamericana editores.

Morris, V. P., David (Editors). (2003). The Arts in a New Millennium: research an the Arts Sector. Westport, CT: Praeger.

Urquiza, A., et al. (2017). Aplicar una distinción. Un programa sistémico-constructivista para la investigación social cualitativa. Revista MAD / Magister en Análisis Sistémico Aplicado a la Sociedad, 21-53.

Young, J. O. (2001). Art and Knownledge. London: Routledge. 